\title{
Recent results on open and closed heavy flavor from PHENIX at RHIC
}

Takashi Hachiya* for the PHENIX collaboration

RIKEN BNL Research Center

E-mail: hachiyaercf.rhic.bnl.gov

Heavy quark production in heavy ion collisions has been used as a probe of the strongly coupled quark gluon plasma in two ways. One is the study of the collision centrality and transverse momentum dependence of the modification of open heavy flavor yields in A+A collisions, which provides information about the coupling of the heavy quark to the medium. The other is the study of the modification of heavy quarkonia in A+A collisions, which provides information about the effect of the medium on the binding of these closed heavy flavor mesons. PHENIX has the ability to study both probes at midrapidity and at forward/backward rapidity. Recently, measurements of quarkonia production in $p(d)+\mathrm{A}$ collisions have shown strong modification of the more weakly bound quarkonia, at the same time that evidence of the formation of a small QGP in these light systems has been found. This proceedings presents results of measurements of separated charm and bottom open heavy flavor modification in $\mathrm{Au}+\mathrm{Au}$ collisions, and charmonia production in $p+\mathrm{Al}$ and $p+\mathrm{Au}$ collisions.

38th International Conference on High Energy Physics

3-10 August 2016

Chicago, USA

${ }^{*}$ Speaker. 


\section{Introduction}

Heavy quarks, bottoms and charms are clean probes to study the nature of strongly coupled quark gluon plasma (QGP) created in relativistic high energy heavy ion collisions. Heavy quarks are mainly produced via initial hard scattering in the collisions because of their large masses $\left(M_{b} \approx\right.$ $4.2 \mathrm{GeV} / c^{2}$ and $M_{c} \approx 1.3 \mathrm{GeV} / c^{2}$ ). Once produced, heavy quarks do not change their flavors and interact with QGP. Then, they suffer the QGP effects such as energy loss, flow effect and dissociation of their pairs. Therefore, the modification of the heavy quark production is sensitive to the property of QGP. Heavy quarks are also modified by cold nuclear matter (CNM) effects. To understand QGP effects and disentangle from CNM effects, it is necessary to measure heavy quarks in $p+p, p+A$ and $A+A$ collisions.

PHENIX measured a variety of open and closed heavy favor productions in $p+p$ and $\mathrm{Au}+\mathrm{Au}$ collisions. We previously measured a strong suppression of the heavy quark production at high transverse momentum $\left(p_{T}\right)$ and a substantial flow in the measurement of single electrons from semi-leptonic decays of bottom and charm hadrons[1,2]. The results are not understood by the expectation of energy loss via gluon radiation such as the dead cone effect[3]. PHENIX $\psi(2 S)$ measurement in central d+Au collisions at mid-rapidity showed more suppression than the $J / \psi$ but similar suppression in peripheral collisions[4]. The sequential suppression of $\psi(2 S)$ and $J / \psi$ can not be explained by the nuclear absorption.

A Barrel and Forward Silicon Vertex Tracker (VTX and FVTX) were installed in PHENIX in 2011 and 2012. VTX (FVTX) covers $|y|<1.2(1.2<|y|<2.2)$ in rapidity and almost $2 \pi$ (full) in azimuth. These silicon detectors add a capability of precise displaced tracking and improves momentum resolution. The precise tracking enables us to separate the bottom and charm components by using the difference of these decay lengths. Better mass resolution allows to separate $J / \psi$ and $\psi(2 S)$ in the di-lepton measurement from their decays. In this article, we report on separated bottom and charm yield in mid and forward rapidity in heavy ion collisions and $J / \psi$ and $\psi(2 S)$ production in $p+\mathrm{A}$ collisions.

\section{Separated bottom and charm yields in Au + Au collisions}

We separate bottom and charm components in the single electron measurement by using the difference of the decay lengths of $B$ and $D$ mesons $\left(c \tau_{B^{0}} \approx 455 \mu \mathrm{m}\right.$ and $\left.c \tau_{D^{0}} \approx 123 \mu \mathrm{m}\right)$. VTX measures the distance of closest approach $(D C A)$ of electrons from the collision vertex. The DCA distribution of inclusive electrons contains a large amount of backgrounds that originate from nondesired physics processes such as Dalitz decays of $\pi^{0}$ and $\eta$, photon conversions as well as detector effects such as mis-identified hadrons and high multiplicity backgrounds. Those backgrounds are estimated and subtracted using the event driven method and the PHENIX detector simulation. The remaining heavy flavor signals from bottoms and charms in the DCA distribution are separated by the unfolding method based on Bayesian inference technique. The analysis procedure is described in detail[5].

The fraction of electrons from bottom decays to inclusive heavy flavors (both bottoms and charms) is shown in the left panel of Fig. 1. The red curve and pink band represent the central value and systematic uncertainties. The fraction is compared with the $p+p$ results $[6,7]$ and FONLL 

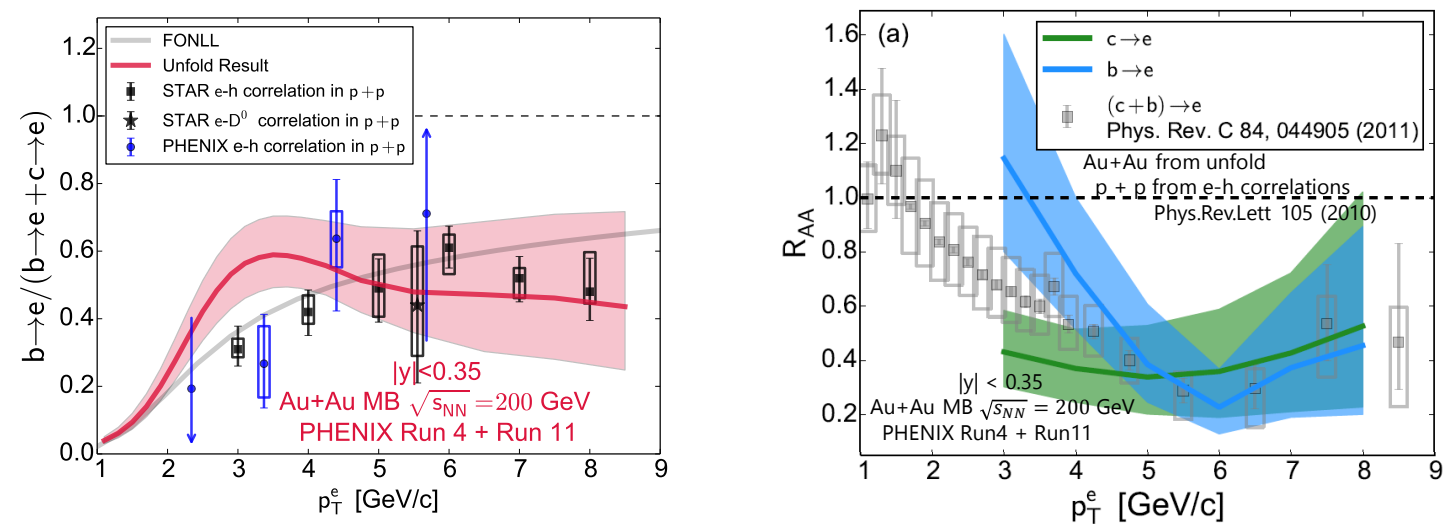

Figure 1: (Left) The unfolded result of bottom electron fraction in minimum bias $\mathrm{Au}+\mathrm{Au}$ collisions at $\sqrt{s_{N N}}=200 \mathrm{GeV}$ [5], which are compared with the FONLL calculation[8]. Also shown are the bottom electron fraction in $p+p$ collisions at $\sqrt{s}=200 \mathrm{GeV}$ from PHENIX[6] and STAR[7]. (Right) $R_{A A}$ for electrons from separated bottom and charm hadron decays as well as inclusive heavy flavor electrons.

perturbative QCD calculation[8] and is found that the $\mathrm{Au}+\mathrm{Au}$ result shows a apparent peak at $p_{T} \sim 3 \mathrm{GeV} / c$ even though $p+p$ results increase monotonically with $p_{T}$ and is well described by the FONLL within uncertainties.

The nuclear modification factor $\left(R_{A A}\right)$ for electrons from bottom and charm hadron decays are calculated using the bottom electron fraction in $\mathrm{Au}+\mathrm{Au}$ and $p+p$, and $R_{A A}$ of inclusive heavy flavor electrons[2], as shown in the right panel of Fig. 1. We find that electrons from bottom decays are less suppressed than that from charm decays for $p_{T}<4 \mathrm{GeV} / c$ and similarly suppressed for higher $p_{T}$. This behavior is qualitatively consistent with the expectations from the mass dependence of energy loss[3,9].

\section{Open bottom measurement from $B \rightarrow J / \psi$ decays in $\mathrm{Cu}+$ Au collisions}

$J / \psi$ from $B$ meson decays is a direct channel to measure open bottom production. PHENIX first measured the signal via $B \rightarrow J / \psi \rightarrow \mu \mu$ at forward and backward rapidity in $200 \mathrm{GeV} \mathrm{Cu}+\mathrm{Au}$ collisions[10]. $J / \psi$ is reconstructed from unlike sign di-muon pairs. FVTX measures DCA of single muons from $J / \psi$ decays. The DCA distribution shows an asymmetric shape for $B$ decays because the negative side of the DCA has better detector acceptance, while prompt $J / \psi$ from the collision vertex shows a symmetric DCA distribution. The different shape of the DCA distribution is used to identify muons from the $B \rightarrow J / \psi$ decays. To extract the $B$ decay signal, the DCA distribution is fit with the template shapes of $B \rightarrow J / \psi$ and prompt $J / \psi$ components determined by PYTHIA + PHENIX detector simulation.

The left panel of Fig. 2 shows the fraction of $B \rightarrow J / \psi$ decays relative to inclusive $J / \psi$ as a function of rapidity in $\mathrm{Cu}+\mathrm{Au}$ and $p+p$ collisions. The fraction in $p+p$ collisions is compared with the FONLL calculation for $B \rightarrow J / \psi[11]$ and Color Evaporation Model for the prompt $J / \psi$ productions[12], and found that the calculation is in agreement with the data within uncertainty. In $\mathrm{Cu}+\mathrm{Au}$ collisions, the fraction is consistent for positive and negative rapidity which correspond to $\mathrm{Cu}$ going and $\mathrm{Au}$ going direction, and higher than that in $p+p$ collisions. The nuclear modification 

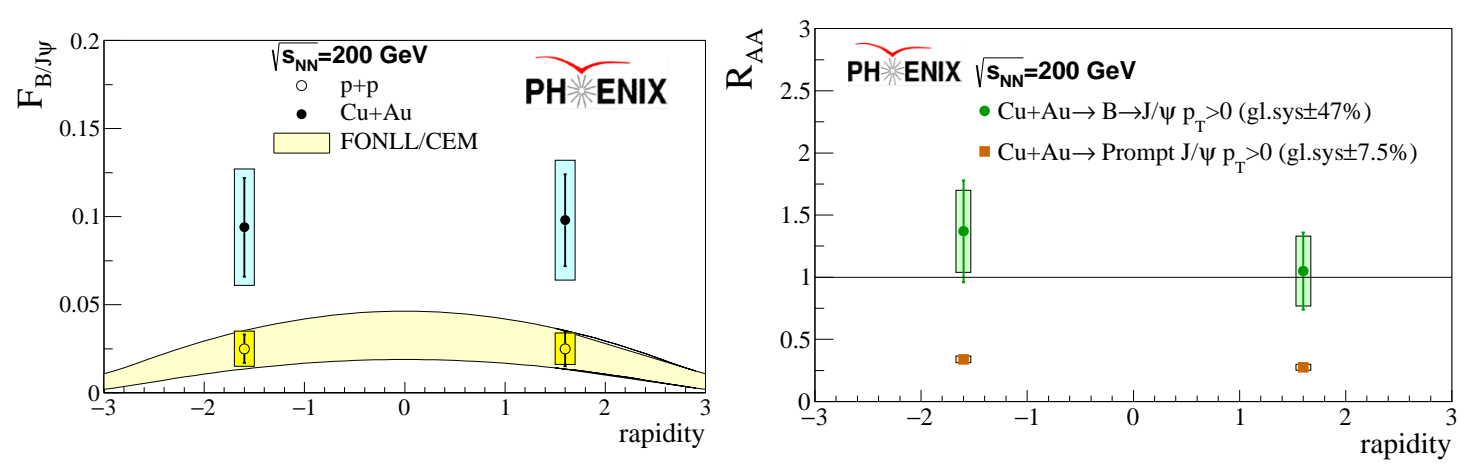

Figure 2: (Left) The fraction of $B \rightarrow J / \psi$ relative to inclusive $J / \psi$ in minimum bias $\mathrm{Cu}+\mathrm{Au}$ collisions at $\sqrt{s_{N N}}=200 \mathrm{GeV}$. (Right) $R_{C u A u}$ for $B \rightarrow J / \psi$ and prompt $\mathrm{J} / \psi$.

factors $R_{C u A u}$ of $B \rightarrow J / \psi$ and prompt $J / \psi$ are calculated using the fraction in $\mathrm{Cu}+\mathrm{Au}$ and $p+p$ collisions and the $R_{C u A u}$ of inclusive $J / \psi$. The right panel of Fig. 2 shows $R_{C u A u}$ of $B \rightarrow J / \psi$ and prompt $J / \psi$. The $R_{C u A u}$ of $B \rightarrow J / \psi$ are consistent with unity within uncertainties for both $\mathrm{Cu}$ and Au going direction. This result indicates that the bottom suffers no suppression within uncertainty while prompt $J / \psi$ is strongly suppressed.

\section{Closed heavy flavor in $\mathbf{p}+$ A collisions : $J / \psi$ and $\psi(2 \mathbf{S})$}

The $J / \psi$ and $\psi(2 \mathrm{~S})$ production in $p+$ A collisions are a good tool to study the CNM effect. The initial state CNM effects such as gluon shadowing and Cronin effect should be similar for both $J / \psi$ and $\psi(2 S)$, while the final state CNM effects such as nuclear breakup effect are different because of their binding energies. PHENIX measures the relative yield of $J / \psi$ and $\psi(2 S)$ in 200 $\mathrm{GeV} p+p, p+\mathrm{Al}$ and $p+\mathrm{Au}$ collisions at forward and backward rapidity.

The double ratio of $\psi(2 \mathrm{~S})$ to $J / \psi$ in $p+\mathrm{A}$ collisions to the same ratio in $\mathrm{p}+\mathrm{p}$ collisions is used to quantify the modification of the production by the CNM effect. Figure 3 shows the preliminary results of the double ratio in $p+\mathrm{Al}$ and $p+\mathrm{Au}$ collisions. The previous result at mid-rapidity in $d+\mathrm{Au}$ collisions is also plotted for the comparison. We find that the double ratio at forward rapidity is consistent with unity within uncertainty. This indicates that the nuclear modifications at forward rapidity are comparable for $J / \psi$ and $\psi(2 \mathrm{~S})$. On the other hand, the double ratio at backward rapidity shows $\psi(2 \mathrm{~S})$ is more suppressed than $J / \psi$ with a factor of 2 . With the result at mid rapidity in $d+$ Au collisions, The double ratio increase linearly with rapidity. The model including the interaction with the co-moving hadrons[13] is compared, and found that the model qualitatively describes the result.

\section{Summary}

PHENIX measured open and closed heavy flavor productions in $\mathrm{Au}+\mathrm{Au}, \mathrm{Cu}+\mathrm{Au}, p+\mathrm{A}$ collisions. VTX and FVTX with excellent performance enables us to separate the bottom and charm components in the single electron measurement in Au+Au collisions and to identify $J / \psi$ from $B$ meson decays in $\mathrm{Cu}+\mathrm{Au}$ collisions. The better mass resolution by FVTX provides the separation of $J / \psi$ and $\psi(2 \mathrm{~S})$ in $\mathrm{p}+\mathrm{A}$ collisions. For the first time, we found the less suppression of electrons 


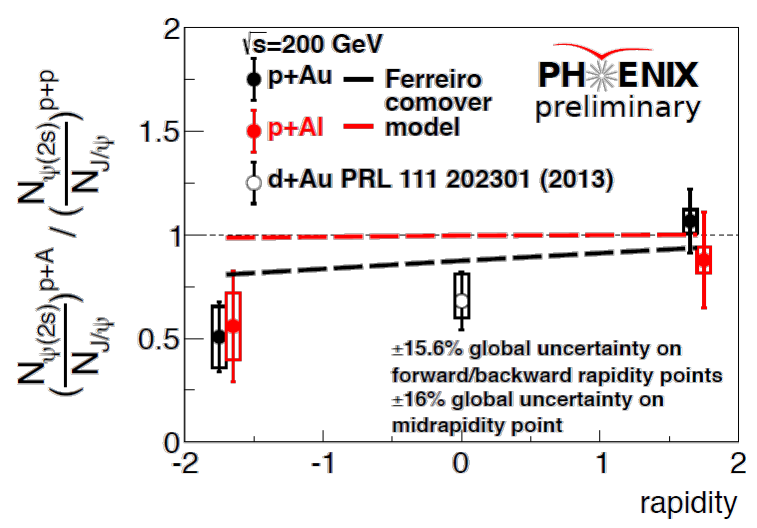

Figure 3: The double ratio of $\psi(2 \mathrm{~S})$ to $J / \psi$ in $200 \mathrm{GeV} p+\mathrm{Al}$ and $p+\mathrm{Au}$ collisions. The model calculation qualitatively describe the data.

from bottom decays than that from charm decays for $3<p_{T}<4 \mathrm{GeV} / \mathrm{c}$, and similar suppression for higher $p_{T}$. The first result of $B \rightarrow J / \psi$ decays also shows the small suppression at low $p_{T}$ in $\mathrm{Cu}+\mathrm{Au}$ collisions. The sequential suppression of $\psi(2 \mathrm{~S})$ and $J / \psi$ is found at backward rapidity, which is described by the comoving hadron model. These results added different dimension to study the QGP property and the CNM effect. We collected large amount of $\mathrm{Au}+\mathrm{Au}$ and $p+p$ data in 2014 to 2016. These new dataset should provide definitive measurements of open and closed heavy flavor productions.

\section{References}

[1] A. Adare et al. Energy Loss and Flow of Heavy Quarks in Au $+\mathrm{Au}$ Collisions at $\sqrt{s_{N N}}=200 \mathrm{GeV}$ Phys. Rev. Lett. 98172301 (2007)

[2] A. Adare et al. Heavy-quark production in $p+p$ and energy loss and flow of heavy quarks in $\mathrm{Au}+\mathrm{Au}$ collisions at $\sqrt{s_{N N}}=200 \mathrm{GeV}$ Phys. Rev. C 84, 044905 (2011)

[3] Y. L. Dokshitzer and D. E. Kharzeev Phys. Lett. B 519, 199 (2001)

[4] A. Adare et al. Nuclear Modification of $\Psi^{\prime}, \chi_{c}$, and $J / \Psi$ Production in d+Au Collisions at $\sqrt{s_{N N}}=$ 200âĂL'âĂL'GeV Phys. Rev. Lett. 111, 202301 (2013)

[5] A. Adare et al. Single electron yields from semileptonic charm and bottom hadron decays in $\mathrm{Au}+\mathrm{Au}$ collisions at $\sqrt{s_{N N}}=200 \mathrm{GeV}$ Phys. Rev. C 93034904 (2016)

[6] A. Adare et al. Measurement of Bottom versus Charm as a Function of Transverse Momentum with Electron-Hadron Correlations in $p+p$ Collisions at $\sqrt{s}=200 \mathrm{GeV}$ Phys. Rev. Lett. 103082002 (2009)

[7] M. Aggarwal et al. Measurement of the Bottom contribution to non-photonic electron production in $p+p$ collisions at $\sqrt{s}=200 \mathrm{GeV}$ Phys. Rev. Lett. 105202301 (2010)

[8] M. Cacciari, P. Nason, and R.Vogt, QCD Predictions for Charm and Bottom Quark Production at RHIC Phys. Rev. Lett. 95122001 (2005)

[9] A. Adil and I. Vitev Collisional dissociation of heavy mesons in dense QCD matter Phys. Lett. B 649 139âĂŞ146 (2007) 
[10] C. Aidala et al., B-meson nuclear modification at forward and backward rapidity in $\mathrm{Cu}+\mathrm{Au}$ collisions at $\sqrt{s_{N N}}=200 \mathrm{GeV}$, arXiv:1702.01085

[11] M. Cacciari, M. Greco, and P. Nason, The $p_{T}$ Spectrum in Heavy-Flavour Hadroproduction, JHEP 05, 007 (1998)

[12] A. D. Frawley, T. Ullrich, and R. Vogt, Heavy flavor in heavy-ion collisions at RHIC and RHIC II, Phys. Rept. 462,790 125 (2008)

[13] E. G. Ferreiro, Excited charmonium suppression in protonâĂŞnucleus collisions as a consequence of comovers, Phys. Lett. B 749 98âĂŞ103 (2015) 